Escuela de Ciencias Sociales y Humanidades, UNED, C.R. URL: http://investiga.uned.ac.cr/revistas/index.php/espiga/index ISSN: 1409-4002 • e-ISSN: 2215-454X

Doi: http://dx.doi.org/10.22458/re.v17i36.2243

\title{
Desarrollo Sostenible para resguardar la seguridad humana, a partir de los resultados del Î́ndice de Progreso Social y su vinculación con la Economía Social Solidaria: Caso Costa Rica
}

\author{
Monserrat Espinach-Rueda* \\ (iD https://orcid.org/0000-0002-2681-4884
}

Recibido: 31 de octubre, 2017 - Aceptado: 16 de agosto, 2018

\section{RESUMEN}

El desarrollo sostenible busca resguardar la seguridad humana a partir de crear progreso social en las comunidades al implementar una Economía Social Solidaria (ESS). Para determinar el avance o rezago de una comunidad o país en seguridad humana, se requiere indicadores que midan el progreso social y, bajo esa filosofía, nace el Índice de Progreso Social (IPS).

A nivel mundial, Costa Rica ha crecido en el IPS comparado con otras naciones, pero al hacer un análisis interno de las mediciones que se dan en 81 cantones del país en el 2017, los resultados visualizan una brecha social dentro de distintas comunidades del país que no permite crecer en condiciones de equidad en seguridad humana para todo el país.

Palabras clave: economía, índice, progreso, seguridad humana.

\section{Formato de citación según APA}

Espinach-Rueda, M. (2018). Desarrollo Sostenible para resguardar la seguridad humana, a partir de los resultados del Índice de Progreso Social y su vinculación con la Economía Social Solidaria: Caso Costa Rica. Revista Espiga 17(36), 159-175. Doi: http:// dx.doi.org/10.22458/re.v17i36.2243

\section{Formato de citación según Chicago-Deusto}

Espinach-Rueda, Monserrat. «Desarrollo Sostenible para resguardar la seguridad humana, a partir de los resultados del Índice de Progreso Social y su vinculación con la Economía Social Solidaria: Caso Costa Rica». Revista Espiga. 17, n. 36 (juliodiciembre, 2018): 159-175. Doi: http://dx.doi.org/10.22458/re.v17i36.2243

* Licenciada en Administración de Empresas con énfasis en Producción de la Universidad Estatal a Distancia, Costa Rica. Maestría en Dirección de Empresas con énfasis en Negocios Internacionales y maestría en Banca y Finanzas, ambas de la Universidad Fundepos, Costa Rica. Especialista en estudios prospectivos. Doctoranda en Ciencias Económicas y Administrativas en la Universidad para la Cooperación Internacional, México. Docente, escritora e investigadora del área de Ciencias de la Administración, Universidad Estatal a Distancia, Costa Rica. Correo: mespinach@uned.ac.cr 


\section{Introducción}

La implementación de la Agenda 2030 de desarrollo sostenible promulgada por la Organización de las Naciones Unidas en el 2015, es una agenda basada en 17 objetivos que fundamenta sus principios en los pilares de resguardo de la naturaleza y de las personas a partir de la Economía Social Solidaria (ESS), la cual nace como una teoría de sostenibilidad que busca un equilibrio entre la responsabilidad social corporativa, el desarrollo humano y una gestión ambiental en armonía con la naturaleza; la finalidad es lograr combatir la pobreza extrema a partir de prácticas solidarias que permitan trabajar en comunidades en alianzas con el gobierno en busca de un desarrollo sostenible ${ }^{1}$.

Los líderes de economías neoliberales han comprendido que ante un calentamiento global sin precedentes producto de la quema de fósiles, el modelo económico actual atenta, a futuro, la existencia de la humanidad ${ }^{2}$. Por consiguiente, el modelo de economía social y solidaria busca, a partir de un comercio justo y un desarrollo sostenible, enmendar el daño causado y crear una economía nueva que permita un desarrollo social de inclusión y equitativo para todas las personas.

La ESS creó las bases para una sensibilización social a partir de la implementación de los principios del desarrollo sostenible que sustenta las bases para una nueva economía ecológica-social solidaria, apoyada por organismos como el Instituto de Investigación de las Naciones Unidas para el Desarrollo Social. Dichos fundamentos sustentan el nuevo modelo económico para seguir impulsado el freno al calentamiento global, eliminar la pobreza y crear comunidades solidarias entre sí, en las cuales el gobierno y las alianzas estratégicas entre organismos internacionales y propios de cada país, involucren a comunidades a través del gobierno local y empresas, con la finalidad de desarrollar un progreso en igualdad de condiciones sociales, ambientales y económicas para todas las personas, que permitan resguardar la seguridad humana ${ }^{3}$.

La importancia de enfocarse en una seguridad humana preventiva implica tener, desde los gobiernos de los países, programas que permitan contemplar a futuro planes de acción que aminoren los efectos de malas prácticas sociales, económicas y ambientales que han acarreado los problemas actuales que vive la sociedad y afectan el bienestar del ser humano. A partir de la necesidad de resguardar la seguridad humana y con la finalidad de medir en el tiempo el logro de alcanzar los objetivos de desarrollo sostenible, nace el Índice de Progreso Social (IPS), compuesto de una serie de indicadores que permite medir las necesidades humanas fundamentales de las comunidades y crear parámetros para dar seguimiento al progreso social en miras de proteger la seguridad humana actual y de las futuras generaciones, todo vinculado al cumplimiento de los 17 Objetivos de Desarrollo Sostenible (ODS), Agenda $2030^{4}$.

1. Naciones Unidas, «La Economía Social y Solidaria y el reto del Desarrollo Sostenible» (2014), acceso: 23 de junio de 2018, http://unsse.org/wp-content/uploads/2014/08/Position-Paper_TFSSE_Esp1.pdf

2. Naciones Unidas, «La devastación de los pobres del mundo: El cambio climático amenaza los avances logrados en materia de desarrollo» (2017), acceso: 20 de junio de 2018, https://unchronicle.un.org/es/article/ la-devastaci-n-de-los-pobres-del-mundo-el-cambio-clim-tico-amenaza-los-avances-logrados-en

3. Naciones Unidas, «La Economía Social...

4. Michael E. Porter y Scott Sterm, «Social Progress Imperative 2017» (2017), acceso: 10 de setiembre de 2018, https://www.df.cl/noticias/site/artic/20170621/asocfile/20170621132906/spanish_2017_social_progress_index_report.pdf 


\section{Objetivo de estudio}

Determinar el grado de resguardo en seguridad humana de Costa Rica, a partir de los resultados de los indicadores de necesidades humanas básicas, fundamentos de bienestar y oportunidades del Índice de Progreso Social.

\section{Método}

Bajo un enfoque cualitativo, a partir de una revisión bibliográfica de documentos emitidos por organizaciones nacionales e internacionales, se hace un análisis contextual de los objetivos de desarrollo sostenible promulgados por la Organización de las Naciones Unidas, externalizado en el IPS en factores de medición como seguridad alimentaria, educación, salud, desarrollo social en armonía con la naturaleza e igualdad de oportunidades para las personas; que permita resguardar la seguridad humana y se complementa con datos provenientes del Estado de la Nación de Costa Rica que validen la información emitida.

\section{Economía Social Solidaria, base fundamental del desarrollo sostenible}

La Economía Social Solidaria es base fundamental del desarrollo sostenible junto con la economía clásica y la economía ambiental; consiste en crear desarrollo «en sus pilares económico, social, ambiental y de empoderamiento comunitario» ${ }^{5}$ a partir de un comercio justo donde se priorice una nueva economía fundamentada en dar «prioridad a las necesidades humanas», «respeto y reconstrucción del medio ambiente» y «control local descentralizado $\rangle^{6}$ enfocados en desarrollar comunidades organizadas que practiquen solidaridad, inclusión social e igualdad de oportunidades para todas las personas ${ }^{7}$.

El sistema actual económico de la mayoría de los países tiene altos déficits presupuestarios y raquíticos crecimientos de progreso y no se contemplan resultados positivos para un progreso social de las personas; el cambio de paradigma social y económico refiere «una gama de formas de producción e intercambio» ${ }^{8}$ en el cual se busca tener objetivos económicos, sociales y humanos que sustenten valores éticos, justicia social y de equidad de género y fomente una economía dinámica de protección social del ser humano y de la naturaleza, contemplando un «empoderamiento sociopolítico» ${ }^{9}$ que busca crear, en las personas, modelos participativos de la ciudadanía en la toma de decisiones desde la

5. Coordinadora latinoamericana y del Caribe de pequeños productores y trabajadores de comercio justo y Fair Trade International, «El comercio justo y los objetivos de desarrollo sostenible: un compromiso de todos», acceso: 20 de octubre de 2017, http://base.socioeco.org/docs/ods-comunicad-v4.pdf

6. David Barkin, «Sobre el progreso y cómo medirlo», acceso: 10 de setiembre de 2018, https://www.youtube. $\mathrm{com} /$ watch? $=\mathrm{x} 0$ OzpjmmZKE

7. Javier Andaluz, «La 'respuesta' de la humanidad al cambio climático», revista Información y Debate 73 (31 de mayo de 2017), acceso: 10 de setiembre de 2018, http://www.revistapueblos.org/?p=21879

8. Instituto de Investigación de las Naciones Unidas para el Desarrollo Social, «Potencial y límites de la Economía Social y Solidaria», acceso: 10 de setiembre de 2018, http://base.socioeco.org/docs/01s_-_sse_ event_span_spanish_for_web_.pdf

9. Ibíd. 
gestión local, donde el poder de las mujeres y los hombres es equitativo y la inclusión social de todos los sectores de la sociedad son parte esencial del desarrollo humano, económico y social. «El modelo político y económico genera un modelo de desarrollo, de persona, de cultura, de sociedad $»^{10}$ que debe trascender entre generaciones según el modelo del desarrollo sostenible. La ESS permite crear un nuevo sistema económico que parte de una vinculación de la responsabilidad social, responsabilidad ambiental, inclusión y equidad en las personas; requiere políticas gubernamentales enfocadas a buscar una autonomía y descentralización de la gobernanza, solidaridad y bienestar humano en las comunidades, para que aprendan a trabajar en equipo, con una diversificación de la producción, desarrollando innovación y ambientes sostenibles con la naturaleza que permitan resguardar la seguridad humana para futuras generaciones ${ }^{11}$.

La ESS busca encontrar formas alternativas para desarrollar un progreso social. Para lograrlo, se requiere un control, tener indicadores de desarrollo humano en términos de salud, nutrición y reducción de carga de factores negativos hacia el medio ambiente para poder llevar mediciones que contemplen planes de acción por seguir según los resultados obtenidos; la finalidad es desarrollar una sensibilización de un cambio de mentalidad de los políticos que gobiernan ${ }^{12}$.

\section{Resguardo de la seguridad humana, finalidad del desarrollo sostenible}

El resguardo de la seguridad humana busca mitigar los problemas de «pobreza, migraciones indocumentadas, tráfico de drogas ilícitas, violaciones a los derechos humanos, regímenes autoritarios, proliferación de armas de destrucción en masa, terrorismo, enfermedades infecciosas y deterioro ambiental ${ }^{13}$. Desde la perspectiva del Programa de Naciones Unidas, resguardar la seguridad humana implica la protección de las personas de las amenazas a sus derechos, su seguridad y su vida; ello engloba procurar la seguridad del individuo en distintas dimensiones como la económica, alimentaria, de salud, ambiental, personal, comunitaria y política, para alcanzar una estabilidad duradera ${ }^{14}$.

Según las Naciones Unidas, «la seguridad humana subraya la necesidad de contar con una nueva estructura que combine los programas de paz y seguridad, desarrollo y derechos humanos de manera más eficaz, eficiente y orientada a la prevención» ${ }^{15}$. Las

10. María Loreto Marcos de la Pisa, «La participación como herramienta de empoderamiento en procesos de inclusión social». Tesis de grado. Universidad de Valladolid, 2012. Acceso: 10 de setiembre de 2018, https://uvadoc.uva.es/bitstream/10324/2646/1/TFG-L\%20117.pdf

11. Blanca Lemus y David Barkin, «Rethinking the Social and Solidarity Economy in Light of Community Practice», United Nations Research Institute for Social Development (2013), acceso: 10 de setiembre de 2018. http://www.unrisd.org/80256B42004CCC77/(httpInfoFiles)/3262F14EE2B6C78AC1257B72003440 26/\$file/Lemus\%20and\%20Barkin\%20draft\%20paper.pdf

12. David Barkin, «Sobre el progreso...

13. Leticia Leal Moya, «Seguridad Humana. La responsabilidad de proteger» (2012):1119, acceso: 30 de agosto de 2018, http://www.revistas.unam.mx/index.php/bmd/article/view/10626/9954

14. Leticia Leal. «Seguridad Humana. La responsabilidad de proteger»(2012):1118, acceso: 30 de agosto de 2018, http://www.revistas.unam.mx/index.php/bmd/article/view/10626/9954

15. Naciones Unidas, «La seguridad humana en las Naciones Unidas» (2012): 3, acceso: 30 de agosto de 2018, https://www.unocha.org/sites/dms/HSU/Outreach/12-41684_Spanish\%20HUMAN\%20SECURITY\%20 Brochure_web_December\%2010.pdf 
características que componen la seguridad humana son «universalidad, interdependencia de sus componentes, centrada en las personas (...) mediante la prevención temprana y [contempla] siete ámbitos relacionados: seguridad económica, alimentaria, de la salud, del medio ambiente, personal, comunitaria y política ${ }^{16}{ }^{16}$ los tres componentes de la seguridad humana son la libertad de vivir sin temor, sin miseria y con dignidad, donde las personas disfrutan de todas las oportunidades de potencializarse como ser humano, disfrutando de todos sus derechos.

\section{Agenda 2030: 17 Objetivos de Desarrollo Sostenible (ODS), base fundamental para resguardar la seguridad humana}

La Organización de las Naciones Unidas (ONU) busca un nuevo orden mundial a partir de la evaluación de la ESS que contemple «procesos de desarrollo que buscan ser inclusivos, sostenibles y justos» ${ }^{17}$. Bajo esas premisas, y a través de acuerdos en materia ambiental y social, se han estipulado distintos acuerdos entre países, en el tiempo que permitan el resguardo de la seguridad humana; así nace la Agenda 2030: Objetivos del Desarrollo Sostenible, base fundamental del IPS que permite desarrollar una economía social solidaria. La clave del éxito del programa de la ONU depende de la gestión política que desarrolle cada territorio del país para cumplir con los objetivos propuestos, la cual contempla cambios en el desarrollo económico actual porque «la sociedad y los políticos tomaron conciencia de que algo falló en la operatividad del modelo económico neoliberal» ${ }^{18}$. La promulgación de los objetivos de desarrollo sostenible es una forma de buscar cambiar el modelo económico actual.

El desarrollo sostenible es el «desarrollo que es capaz de satisfacer las necesidades actuales sin comprometer los recursos y posibilidades de las futuras generaciones, eliminando la pobreza y promoviendo la equidad social» ${ }^{19}$ a partir de implementar en cada territorio los 17 ODS, que contemplan 169 metas para resguardar la seguridad humana; se crea un progreso sin crear daño ambiental que busca erradicar la pobreza a través de la inclusión social de todas las personas en la sociedad ${ }^{20}$.

Los ejes de acción del desarrollo sostenible son: personas, planeta, prosperidad, paz y alianzas que permitan agrupar «tareas y áreas que pueden ser consideradas independientes y aisladas entre sí ${ }^{21}$, que permitan un crecimiento inclusivo, equitativo, innovador y productivo para resguardar la naturaleza y la seguridad humana. Para medir el

16. Naciones Unidas, «La seguridad humana en las Naciones Unidas» (2012): 7, acceso: 30 de agosto de 2018, https://www.unocha.org/sites/dms/HSU/Outreach/12-41684_Spanish\%20HUMAN\%20SECURITY\%20 Brochure_web_December\%2010.pdf

17. Instituto de Investigación de las Naciones Unidas para el Desarrollo Social, «Potencial y límites..

18. Lissette Bustillo García y Juan Pablo Martínez Dávila, «Los enfoques del desarrollo sustentable», Interciencia, Vol. 33, n. ${ }^{\circ} 5$ (2008): 389.

19. Pedro César Cantú Martínez, «El axioma del desarrollo sustentable», Revista de Ciencias Sociales, Vol. 3 , n. ${ }^{\circ} 134$ (2012): 88

20. Lissette Bustillo García y Juan Pablo Martínez Dávila, «Los enfoques del..., 389.

21. Pablo José Martínez Osés, «Un análisis del poder en la Agenda 2030: oportunidades para un cambio de paradigma de desarrollo», Economistas sin Fronteras, dossieres EsF, n. ${ }^{\circ} 26$ (2017): 11, acceso: 10 de setiembre de 2018, http://base.socioeco.org/docs/dossieres-esf-26-repensando-el-modelo-de-sociedad-yde-econom_c3_ada.pdf 
campo de acción de los ejes del desarrollo sostenible, nace el IPS como una forma de medir indicadores que resguarden la seguridad humana; estos contemplan el cumplimiento de los 17 ODS, Agenda 2030 aprobada en el Pacto de París en 2015 por 197 países que integran la $\mathrm{ONU}^{22}$.

Los objetivos del desarrollo sostenible son «un agregado de demandas transnacionales, reflejo de las tensiones actualmente existentes entre los diferentes actores y sus distintas visiones del desarrollo» ${ }^{23}$. Por tanto, la creación del IPS permite medir el progreso social de un país bajo las premisas del desarrollo sostenible ${ }^{24}$.

\section{Aspectos generales del Índice de Progreso Social que permiten medir economías enfocadas en desarrollo sostenible}

El modelo del IPS estima el progreso social-económico y ambiental de un país o comunidad a partir de la medición de los indicadores que lo conforman. La finalidad es determinar fortalezas y debilidades del sistema económico de un país en desarrollo sostenible y comparar los resultados con el avance que se tiene anualmente como país. «El progreso social es la capacidad de una sociedad de satisfacer las necesidades humanas fundamentales de sus ciudadanos, de establecer cimientos que permitan mejorar y mantener la calidad de vida de sus ciudadanos y comunidades, y de crear las condiciones para que todos los individuos alcancen su pleno potencial» ${ }^{25}$.

Para medir las distintas variables, el IPS incorpora en su diseño cuatro aspectos importantes para medir el progreso de una nación, a través de indicadores que miden el bienestar social de las personas e indicadores ambientales que buscan el desarrollo sostenible de las personas para resguardar la seguridad humana y de la naturaleza (ver tabla 1).

TABLA 1

Diseño para medir el progreso de una nación

\begin{tabular}{ll}
\multicolumn{1}{c}{ Diseño } & \multicolumn{1}{c}{ Medición } \\
Indicadores sociales y ambientales & $\begin{array}{l}\text { Analizar la relación de desarrollo económico medido por el PIB per } \\
\text { cápita y el desarrollo social }\end{array}$ \\
Objetivo & Medir los resultados importantes para la seguridad humana \\
Progreso social holístico & $\begin{array}{l}\text { Medir de forma integral la salud de las sociedades basado en el grado de } \\
\text { cumplimiento de los objetivos de desarrollo sostenible }\end{array}$ \\
Herramienta de aplicación & $\begin{array}{l}\text { A partir de los resultados y clasificación, se provee una calificación país } \\
\text { que le permite mejorar cada año }\end{array}$
\end{tabular}

Fuente: Elaboración propia con base en Porter y Sterm, 2017.

22. United Nations, Sustainable Development Goals. 17 Goals to transform our world (s.f.), acceso: 10 de setiembre de 2018, http://www.un.org/sustainabledevelopment/

23. César Cantú Martínez, «El axioma del desarrollo sustentable», Revista de Ciencias Sociales, n. ${ }^{\circ} 137$ (2012): 88, acceso: 10 de setiembre de 2018, https://revistas.ucr.ac.cr/index.php/sociales/article/view/8420/7943

24. Porter y Sterm, «Social Progress...»

25. Porter y Sterm, «Social Progress...» 
TABLA 2

Componentes del Índice de Progreso Social

\section{Necesidades humanas básicas}

Nutrición y cuidados humanos básicos

Agua y saneamiento

Vivienda

Seguridad personal
Fundamentos de bienestar

Acceso a conocimientos básicos

Acceso a información y comunicaciones

Salud y bienestar

Calidad medioambiental

\section{Oportunidades}

Derechos personales

Libertad personal y de elección

Tolerancia e inclusión

Acceso a educación superior

Fuente: Scott Sterm, Amy Wares y Sarah Orzell. Índice de Progreso Social 2017. Reporte Metodológico (2017): 9.

El IPS tiene componentes que permiten determinar los indicadores de medición; cada indicador principal (necesidades humanas básicas, fundamentos, oportunidades) se combina con otros indicadores que permiten determinar el progreso de cada variable analizada de forma individual (ver tabla 2).

Los puntajes del IPS tienen una escala de 0 a 100, donde 0 es la escala más baja en progreso social o desarrollo sostenible y 100 la mejor calificación. El IPS se calcula como «el promedio simple de las tres dimensiones» ${ }^{26}$ de necesidades básicas, fundamentos de bienestar y oportunidades. Estos indicadores se descomponen en distintos sub indicadores que conforman el IPS.

TABLA 3

Indicadores de necesidades básicas

\begin{tabular}{ll}
\multicolumn{1}{c}{ Necesidades humanas básicas } & \multicolumn{1}{c}{ Indicador } \\
Nutrición y cuidados médicos básicos & Desnutrición \\
& Intensidad del déficit alimentario \\
& Tasa de mortalidad materna \\
& Tasa de mortalidad infantil \\
& Muertes por enfermedades infecciosas \\
& Acceso a agua potable \\
Agua y saneamiento & Acceso rural a fuentes de agua mejoradas \\
& Acceso a infraestructura de saneamiento mejorado \\
Vivienda & Disponibilidad de vivienda accesible \\
& Acceso a energía eléctrica \\
& Calidad del suministro eléctrico \\
& Muertes atribuibles a la contaminación del aire en los hogares \\
Seguridad personal & Tasa de homicidios \\
& Nivel de crímenes violentos \\
& Criminalidad percibida \\
& Terror político \\
& Muertes en carreteras \\
\end{tabular}

Fuente: Sterm, Wares y Orzell (2017): 9.

26. Scott Sterm, Amy Wares y Sarah Orzell, «Índice de Progreso Social 2017. Informe Metodológico» (2017), acceso: 10 de setiembre de 2018, http://www.socialprogressindex.com/assets/downloads/resources/es/ Spanish-2017-Social-Progress-Index-Methodology-Report.pdf 
TABLA 4

Indicadores de fundamentos de bienestar

\begin{tabular}{ll}
\multicolumn{1}{c}{ Fundamentos de bienestar } & \multicolumn{1}{c}{ Sub indicador } \\
Acceso a conocimientos básicos & Tasa de alfabetización en adultos \\
& Matriculación en educación primaria \\
& Matriculación en educación secundaria a nivel de bachillerato \\
& Paridad de género en educación secundaria
\end{tabular}

Fuente: Sterm, Wares y Orzell (2017): 9.

La tabla 3 detalla los diferentes indicadores que componen al macro indicador de necesidades humanas básicas. Este se descompone en cuatro indicadores (nutrición y cuidados médicos básicos, agua y saneamiento, vivienda, seguridad personal); estos otros indicadores se componen de distintas variables que buscan medir el grado de impacto social en distintos territorios del país.

La tabla 4 detalla los sub indicadores que conforman al macro indicador de fundamentos de bienestar como son: acceso a conocimientos básicos, acceso a información y comunicaciones, salud y bienestar y calidad medioambiental. Las distintas variables miden el grado social que puede tener los territorios a nivel interno entre distintas comunidades o cantones en el caso de Costa Rica, así como a nivel externo cuando se hacen comparaciones entre países.

La tabla 5 detalla la composición del indicador de oportunidades que permite medir el grado de coyuntura que puede tener un territorio en derechos personales, libertad personal y de elección, tolerancia e inclusión y acceso a educación superior.

\section{Resultados del Índice de Progreso Social a nivel global en el 2017}

Para el 2017 se realizó una medición del IPS de 128 países, incluido Costa Rica. La tabla 6 agrupa los resultados obtenidos en distintos niveles de progreso como son: progreso social muy alto, progreso social alto, progreso social medio alto, progreso social medio bajo, progreso social bajo y progreso social muy bajo, con calificaciones de 0 hasta 100 para los distintos países donde la nota 100 implica que es un país $100 \%$ con desarrollo sostenible. 
TABLA 5

Indicadores de oportunidad

\begin{tabular}{|c|c|}
\hline Oportunidades & Sub indicador \\
\hline Derechos personales & $\begin{array}{l}\text { - Derechos políticos } \\
\text { - Libertad de expresión } \\
\text { - Libertad de reunión } \\
\text { - Derecho a la propiedad privada }\end{array}$ \\
\hline Libertad personal y de elección & $\begin{array}{l}\text { - Libertad de decidir sobre su vida } \\
\text { - Libertad de culto } \\
\text { - Matrimonio adolescente } \\
\text { - Satisfacción de la demanda de métodos anticonceptivos } \\
\text { Corrupción }\end{array}$ \\
\hline Tolerancia e inclusión & $\begin{array}{l}\text { - Tolerancia hacia los inmigrantes } \\
\text { - Tolerancia hacia los homosexuales } \\
\text { - Discriminación y violencia hacia las minorías } \\
\text { - Tolerancia religiosa } \\
\text { - Redes de apoyo en la comunidad }\end{array}$ \\
\hline Acceso a educación superior & $\begin{array}{l}\text { - Años de educación terciaria } \\
\text { - Años promedio de escolaridad de las mujeres } \\
\text { - Inequidad en la obtención de educación } \\
\text { - Universidades de rango mundial } \\
\text { - Porcentaje de estudiantes de educación superior matriculados en } \\
\text { universidades de clase mundial }\end{array}$ \\
\hline
\end{tabular}

Fuente: Sterm, Wares y Orzell (2017): 9.

TABLA 6

Clasificaciones del Índice de Progreso Social a nivel global para el año 2017

\begin{tabular}{llcc}
\multicolumn{1}{c}{ Clasificación del Índice de Progreso Social } & \multicolumn{1}{c}{ Principales puestos } & Posición país & Puntaje \\
Progreso social muy alto & Dinamarca & 1 & 90,57 \\
& Finlandia & 2 & 90,53 \\
& Noruega & 3 & 90,27 \\
Progreso social alto & Bélgica & 15 & 87,15 \\
& España & 16 & 86,96 \\
\multirow{2}{*}{ Progreso social medio alto } & Japón & 17 & 86,44 \\
& Mauricio & 39 & 75,18 \\
& Panamá & 40 & 74,61 \\
Progreso social medio bajo & Bulgaria & 41 & 74,42 \\
& El Salvador & 70 & 66,43 \\
Progreso social bajo & Líbano & 71 & 66,31 \\
& Moldavia & 71 & 66,31 \\
Progreso social muy bajo & Kenia & 95 & 56,17 \\
& Myanmar & 96 & 55,69 \\
& Bangladesh & 97 & 54,84 \\
& Yemen & 122 & 43,46 \\
& Guinea & 123 & 43,40 \\
\hline
\end{tabular}

Fuente: Elaboración propia con base en datos de Porter y Sterm (2017): 4-5. 
Costa Rica ocupa el puesto 28 de los 128 países que fueron medidos por el IPS en el 2017; se encuentra dentro de la categoría de Progreso social alto, con una puntuación de 81,03; a nivel latinoamericano es superado solamente por Chile. En Centroamérica, Costa Rica tiene la mejor posición de la región, seguida por Panamá (40), El Salvador (70), Nicaragua (81), Guatemala (84) y Honduras (89) 27.

La mayor relación de desempeño económico de un país se da cuando el crecimiento del producto interno bruto per cápita crece junto al progreso social, aunque el desempeño económico, por sí solo, no explica por completo el progreso social» ${ }^{28}$. Por esta razón, países que crecen levemente en el PIB per cápita y suben en el IPS, implica que realizan grandes avances en desarrollo sostenible y en acciones que permiten buscan el resguardo de la seguridad humana. El crecimiento de Costa Rica en el puntaje del IPS está justificado por el crecimiento constante del PIB per cápita, donde la economía del país, para 2017, creció aproximadamente un 3,8\% anual, ${ }^{29}$ esto permite apoyar mayor cantidad de programas sociales que mejoran la calidad de vida de las personas y se refleja en los resultados del IPS, permitiendo avanzar hacia un mayor desarrollo sostenible en el país.

Las posiciones del IPS entre países varían cada año con respecto de la cantidad de países que son objeto de medición, pero lo que importa es el valor absoluto obtenido en el puntaje de medición. Costa Rica mejoró en 3,28 puntos en el IPS; para el 2014 se posicionaba con un puntaje de 77,75 y para 2017 alcanza un puntaje de 81,03 (ver tabla 7).

TABLA 7

Índice de Progreso Social del año 2014 al 2017 para Costa Rica

\begin{tabular}{cccc} 
Año & Posición & Puntaje & Cantidad de países medidos \\
2014 & 25 & 77,75 & 132 \\
2015 & 28 & 77,88 & 133 \\
2016 & 28 & 80,12 & 133 \\
2017 & 28 & 81,03 & 128 \\
\hline
\end{tabular}

Fuente: Elaboración propia con base en datos de Porte y Sterm (2014), Porte y Sterm (2015), Porte y Sterm (2016) y Porte y Sterm (2017).

Para el cálculo del valor obtenido en el IPS de Costa Rica, se hace un análisis de los 81 cantones que conforman al país en el 2017; se miden distintos indicadores y se ponderan los resultados para determinar el resultado país, que es la base de información conocida a nivel mundial. En Costa Rica, según los indicadores de necesidades humanas, fundamentos del bienestar y oportunidades, hay comunidades que están mejor o peor posicionadas y afectan el IPS general del país. Las mediciones que hace el Ministerio de

27. Porter y Sterm, Índice de Progreso Social 2017, 4-5.

28. United Nations, Sustainable Development Goals, 17 Goals to Transform Our World (s.f.), acceso: 10 setiembre de 2018, http://www.un.org/sustainabledevelopment/

29. Banco Central de Costa Rica, Revisión Programa Macroeconómico 2017-2018 (2017): 3, acceso: 10 de setiembre de 2017, https://www.bccr.fi.cr/seccion-noticias/Noticia/Revision_Programa_2017-2018.aspx 
Planificación Nacional y Política Económica junto con otras instancias ${ }^{30}$, miden a cada cantón de forma individual y permite generar información para desarrollar planes de acción por seguir según los resultados de cada indicador.

La importancia de dar seguimiento al IPS es que identifica las regiones que más ayuda requieran según los datos reflejados en los resultados del IPS. Cuando se busca implementar progreso social, ambiental y económico equitativo para todas las comunidades, es necesario analizar los resultados de los distintos indicadores que permitan tener bases de comparación, tarea que se realiza desde el IPS de cada cantón del país. La tabla 8 presenta los cantones mejor y peor posicionados en Costa Rica según el IPS.

TABLA 8

Cantones mejor y peor posicionados según el IPS

\begin{tabular}{lclc}
\multicolumn{2}{c}{ Cantones mejor posicionados } & \multicolumn{2}{c}{ Cantones peor posicionados } \\
Cantón & IPS (puntaje) & Cantón & IPS (puntaje) \\
Moravia & 81,98 & Talamanca & 61,66 \\
Flores & 81,02 & La Cruz & 63,80 \\
San Rafael de Escazú & 80,78 & Los Chiles & 64,02 \\
Zarcero & 80,55 & Garabito & 65,43 \\
San Isidro de Heredia & 80,38 & Upala & 67,21 \\
\hline
\end{tabular}

Fuente: Espinach (2017): 64-65.

Según la tabla 8, el resultado obtenido por los indicadores que conforman el IPS en Costa Rica presenta como cantones mejor posicionados del país a los de la región central, como es el caso de Moravia (provincia de San José), Flores y San Rafael (provincia de Heredia). Las zonas costeras y limítrofes con Panamá y Nicaragua son los cantones más pobres del país y con el menor IPS; es necesario eliminar esa brecha tan grande entre cantones para mejorar en la calificación país del IPS.

Para implementar el desarrollo sostenible en un país, se utilizan los datos que genera el IPS cantonal; para eso es necesario ejecutar planes de acción y de mejora a partir de identificar los resultados de los cantones más vulnerables y con más baja calificación en el IPS; la información obtenida genera una estrategia de desarrollo en gestión local territorial, con la contemplación de programas que busquen mejorar las acciones que miden las variables que conforman los distintos indicadores. Al realizar planes estratégicos enfocados en resguardar la seguridad humana del país a partir de mejorar los indicadores de medición del IPS, se crea desarrollo sostenible.

Los indicadores que componen el IPS varían en cada comunidad. Hay poblaciones que están mejor posicionadas que otras según el criterio de cada indicador, lo que permite identificar qué planes de acción se están realizando en ciertas comunidades y permite trazar una ruta por seguir para mejorar los indicadores. La finalidad del desarrollo sostenible es que todas las comunidades tengan el mismo IPS. La tabla 9 detalla los mejores

30. Costa Rica Propone, «Costa Rica Propone» (s.f.), acceso: 10 de setiembre de 2018, https://www.costaricapropone.go.cr/ 
cantones del país en los indicadores de necesidades humanas, fundamentos de bienestar y oportunidades que conforman el IPS del país.

TABLA 9

Cantones mejor posicionados en Costa Rica según la composición del IPS

\begin{tabular}{lclclc}
\multicolumn{2}{c}{$\begin{array}{c}\text { Indicador de necesidades } \\
\text { humanas básicas }\end{array}$} & \multicolumn{2}{c}{$\begin{array}{c}\text { Indicador de fundamentos } \\
\text { de bienestar }\end{array}$} & \multicolumn{2}{c}{ Indicador de oportunidades } \\
Cantón & Puntaje & \multicolumn{1}{c}{ Cantón } & Puntaje & Cantón & Puntaje \\
León Cortés & 94,04 & Zarcero & 76,13 & Montes de Oca & 82,61 \\
Palmares & 93,81 & Moravia & 76,05 & Moravia & 80,63 \\
Acosta & 93,49 & Heredia & 75,53 & Flores & 80,52 \\
Santa Bárbara & 92,43 & Hojancha & 75,21 & Belén & 79,91 \\
Barva & 92,33 & Escazú & 74,60 & Escazú & 78,77 \\
\hline
\end{tabular}

Fuente: Espinach (2017): 64.

Según los datos de la tabla 9, en Costa Rica los cantones mejor posicionados tienen indicadores de necesidades humanas básicas con puntajes por arriba de los 90, seguido de los indicadores que componen el macro indicador de «oportunidades» con una media aproximada a un valor de 80 puntos. Los indicadores de «fundamentos de bienestar» en los cantones mejor posicionados, son los que tienen un puntaje más bajo pero en su mayoría se encuentran por arriba de 75 puntos. Una estrategia para crecer en desarrollo sostenible en cantones con altas posiciones, consiste en mejorar las acciones que se miden con los indicadores de fundamentos de bienestar en criterios de acceso al conocimiento básico, acceso a información y comunicaciones, calidad de medio ambiental y el criterio de salud y bienestar.

La tabla 10 detalla los valores de los indicadores que componen el IPS de los cantones más vulnerables en desarrollo sostenible del país.

TABLA 10

Cantones peor posicionados en Costa Rica según la composición del IPS

\begin{tabular}{|c|c|c|c|c|c|}
\hline \multicolumn{2}{|c|}{$\begin{array}{l}\text { Indicador de necesidades } \\
\text { humanas básicas }\end{array}$} & \multicolumn{2}{|c|}{$\begin{array}{l}\text { Indicador de fundamentos } \\
\text { de bienestar }\end{array}$} & \multicolumn{2}{|c|}{ Indicador de oportunidades } \\
\hline Cantón & Puntaje & Cantón & Puntaje & Cantón & Puntaje \\
\hline Talamanca & 62,66 & Osa & 61,60 & Los Chiles & 57,05 \\
\hline Garabito & 63,18 & Orotina & 62,57 & Talamanca & 58,28 \\
\hline La Cruz & 67,43 & Los Chiles & 62,69 & Guatuso & 60,05 \\
\hline Liberia & 69,65 & La Cruz & 62,73 & Matina & 60,87 \\
\hline Limón & 70,99 & Palmares & 62,75 & La Cruz & 61,24 \\
\hline
\end{tabular}

Fuente: Espinach (2017): 65.

Según la tabla 10, los cantones con menor IPS de Costa Rica presentan, en todos los indicadores que componen al IPS, lecturas muy bajas comparadas con los resultados de cantones de la región central. Las estrategias enfocadas en resguardar la seguridad humana de los territorios costeros y fronterizos, deben contemplar acciones de mejora en 
todos los criterios que componen los distintos indicadores, con la finalidad de mejorar en desarrollo sostenible.

\section{Situación socioeconómica de Costa Rica según Estado de la Nación}

En Costa Rica, todos los años, el Gobierno da seguimiento a una serie de indicadores que conforman la base para medir al país en variables como desarrollo humano sostenible, economía, pobreza, equidad social, empleo, fortalecimiento de la democracia, armonía con la naturaleza, sistema electoral y ambiente, variables que permiten identificar el estado actual del país ${ }^{31}$.

Los principales resultados del informe destacan que Costa Rica presenta resultados positivos a mediano plazo, donde «el costo de la canasta básica alimentaria como la línea de pobreza disminuyeron (...), bajó el desempleo; aumentó en términos reales el ingreso total promedio de los hogares $\rangle^{32}$; esto confirma que los resultados del IPS están alineados a los informes que se generan desde el Estado de la Nación.

A pesar de que los resultados en aspectos sociales, económicos y políticos han sido positivos en el 2017, el alto financiamiento de deuda pública del gobierno hace crecer el riesgo de que en el futuro no se pueda mantener una sostenibilidad en desarrollo humano; por consiguiente, los indicadores como los del IPS se podrían ver afectados y tenderían a la baja las mediciones en desarrollo sostenible. Los programas del Gobierno sin una reforma fiscal no serán sostenibles, ya que «el $61 \%$ de la reducción de la pobreza por ingresos se explica por las transferencias de los programas sociales» ${ }^{33}$. El país ha crecido en calidad de vida producto de la alta inversión del Gobierno para fortalecer las poblaciones vulnerables del país.

\section{Situación socioeconómica de la región centroamericana al 2017}

Los países centroamericanos más dinámicos en incremento del Producto Interno Bruto real interanual son Nicaragua y Costa Rica, en cambios porcentuales positivos durante el periodo 2014-2016. El país que más crece cada año es Panamá, pero ha sufrido una contracción en el crecimiento porcentual durante el 2016 con respecto al año anterior (ver figura 1).

A nivel social, variables que miden la calidad de vida en aspectos de mortalidad infantil, necesidades básicas satisfechas, inversiones que generan empleo, bienestar social en salud y educación, exclusión social y aspectos como salario mínimo agrícola que cubra la canasta básica, presentan grandes brechas sociales de desigualdad en Centroamérica. Costa Rica destaca como el país más sostenible socialmente; los demás países de la

31. Estado de la Nación 2017, «Estado de la Nación en Desarrollo Humano Sostenible» (2017), acceso: 10 de setiembre de 2018, https://estadonacion.or.cr/2017/assets/en-23-2017-book-low.pdf

32. Ibíd, 32 .

33. Ibíd, 42 . 


\section{Cambios porcentuales del PIB real para Centroamérica \\ del periodo 2014-2016}

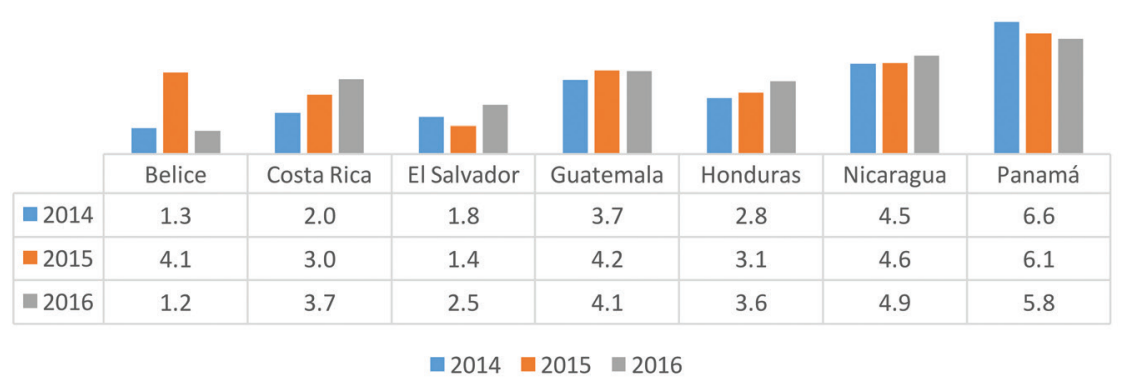

Figura 1. Cambios porcentuales del PIB real para Centroamérica del año 2014 al 2016. Fuente: Elaboración propia con base en datos del Estado de la Región (2016).

región, en diferentes variables de medición, son afectados por la baja inversión de parte de los gobiernos para esos programas ${ }^{34}$.

\section{Conclusión}

Antes de dar prioridad al desarrollo sostenible, el progreso económico era medido con referencia al PIB per capita por habitante de una nación; este no contemplaba aspectos de seguridad humana y social de las personas. Países con altos crecimientos del PIB tenían comunidades desprotegidas en aspectos sociales y humanos para las personas. Ante esa paradoja nació el IPS para medir no solo el PIB per cápita de los países, sino sumarle también indicadores que midan la seguridad humana.

La Economía Social Solidaria, a través del desarrollo sostenible, es la forma de empoderar a las personas, comunidades, gobiernos y empresas, entre otras organizaciones, para que tengan una sensibilización que les permita buscar el bienestar social, humano y ambiental; por tanto, resguardar la seguridad humana implica que las personas tengan calidad de vida y progreso humano y social en armonía con la naturaleza. La seguridad humana consiste en crear expectativas que permitan suplir las necesidades humanas básicas, medir los fundamentos de bienestar y que las personas tengan oportunidades de salir adelante.

A nivel mundial, Costa Rica ha avanzado en crecimiento social de las personas; pero hay mucho que hacer para mejorar como país en desarrollo sostenible que proteja la seguridad humana en todo el territorio. Un desarrollo sostenible debe ser equitativo para todas las comunidades que conforman un país y las desigualdades de necesidades humanas básicas, los fundamentos de bienestar y oportunidades para las personas de distintos cantones de Costa Rica son muy desiguales y poco equitativos.

34. Estado de la región, «Quinto Informe Estado de la Región en Desarrollo Humano Sostenible 2016» (2016), acceso: 10 de setiembre de 2018, https://www.estadonacion.or.cr/erca2016/assets/erca-2016-web.pdf 
El IPS es una medida para valorar el desarrollo sostenible que se realiza en el territorio nacional, identifica los aspectos críticos por mejorar a partir del desarrollo de estrategias que busquen mejorar los resultados actuales de los indicadores, de una forma inclusiva y equitativa en todo el país.

Costa Rica ha construido grandes logros en desarrollo económico social y ambiental. Algunos cantones del país, principalmente los que se ubican en la región central, tienen, según el IPS del 2017, indicadores muy fuertes, reflejados en calidad de vida para las personas; sin embargo, los resultados de cantones costeros y fronterizos presentan una situación muy distinta a lo que viven los cantones de la región central, con brechas significativas en desarrollo humano-social-económico.

Los resultados del IPS de los distintos cantones del país invita a la ciudadanía a reflexionar y buscar alternativas de solución para mejorar la desigualdad social y económica que se vive, principalmente en territorios costeros y fronterizos.

\title{
ABSTRACT
}

Sustainable Development to Safeguard Human Security, Based on the Results of the Social Progress Index and Its Link to Social and Solidarity Economy: The Case of Costa Rica

\begin{abstract}
Sustainable development seeks to safeguard human security by means of creating social progress in communities through Social and Solidarity Economy (SSE). Determining a community's or country's level of human security development requires indicators which measure social progress. The Social Progress Index (SPI) was created with that aim in mind.

When compared with other nations, Costa Rica has grown globally in the SPI. Nevertheless, when an internal analysis of the measurements made in 81 cantons in 2017 is performed, the results reveal a social gap within different communities, which does not allow for equal growth in human security throughout the country.
\end{abstract}

Key words: Economy, index, progress, human security.

RÉSUMÉ

Développement durable pour sauvergarder la sécurité humaine, à partir des résultats de l'Indice de progrès social et son lien avec l'Économie sociale et solidaire (ESS): Le cas du Costa Rica

Le développement durable vise à sauvergarder la sécurité humaine à partir de la création du progrès social dans les communautés et de l'implémentation d'une Économie sociale et solidaire (ESS). Afin de déterminer le progrès ou le retard d'une communauté ou pays en matière de sécurité humaine, il faut des indicateurs pour mesurer le progrès social ; sous cette philosophie est né l'Indice de progrès social (IPS).

Au niveau mondial, Le Costa Rica a obtenu de meilleurs résultats de l'IPS par rapport à d'autres pays. Une analyse interne des mesures dans 81 cantons du pays dans l'année 2017 a mis en évidence la fracture sociale des différentes communautés du pays ce qui ne permet pas le développement dans des conditions d'équité en sécurité humaine pour tous.

Mots-clés: Économie, indice, progrès, sécurité humaine. 


\section{Bibliografía}

Andaluz, Javier «La 'respuesta' de la humanidad al cambio climático». Revista Información y Debate 73 (31 de mayo de 2017). Acceso: 10 de setiembre de 2018. http://www.revistapueblos.org/?p=21879

Banco Central de Costa Rica. Revisión Programa Macroeconómico 2017-2018 (2017): 3. Acceso el 10 setiembre de 2018. https://www.bccr.fi.cr/seccion-noticias/Noticia/Revision_Programa_2017-2018.aspx

Barkin, David. «Sobre el progreso y cómo medirlo». Acceso: 10 setiembre de 2018. https://www.youtube.com/ watch? $=x 0$ OzpjmmZKE

Bustillo García, Lissette y Juan Pablo Martínez Dávila. «Los enfoques del desarrollo sustentable». Interciencia, Vol. 33, n. ${ }^{\circ} 5$ (2008): 389

Cantú Martínez, Pedro César. «El axioma del desarrollo sustentable». Revista de Ciencias Sociales. Universidad de Costa Rica, Vol. 3, n. ${ }^{\circ} 134$ (2012): 88. doi: https://doi.org/10.15517/rcs.v0i137.8420

Costa Rica Propone(s.f.). Acceso: 10 de setiembre de 2018. http://www.costaricapropone.go.cr/ranking?type=IPS

Espinach Rueda, Monserrat. «Agenda 2030 del desarrollo sostenible promulgada por la organización de las Naciones Unidas. Caso Costa Rica». Revista Ágora de heterodoxias. Universidad Centrooccidental Lisandro Alvarado, Vol. 3, n. ${ }^{\circ} 2$ (2017): 50-67.

Estado de la Nación 2017. «Estado de la Nación en Desarrollo Humano Sostenible» (2017). Acceso: 10 de setiembre de 2018. https://estadonacion.or.cr/2017/assets/en-23-2017-book-low.pdf

Estado de la Región. «Estadísticas de Centroamérica 2017» (2017). Acceso: 10 setiembre de 2018. https://www. estadisticascentroamerica2017.estadonacion.or.cr/estadisticas-erca2017.html\#econ\%C3\%B3mico

Estado de la Región. «Quinto Informe Estado de la Región en Desarrollo Humano Sostenible 2016» (2016). Acceso: 10 de setiembre de 2018. https://www.estadonacion.or.cr/erca2016/assets/erca-2016-web.pdf

Coordinadora latinoamericana y del Caribe de pequeños productores y trabajadores de comercio justo y Fair Trade International. «El comercio justo y los objetivos de desarrollo sostenible: un compromiso de todos». Acceso: 10 de setiembre de 2018. http://base.socioeco.org/docs/ods-comunicad-v4.pdf

Instituto de Investigación de las Naciones Unidas para el Desarrollo Social. Potencial y Límites de la Economía Social y Solidaria. Acceso: 10 de setiembre de 2018. http://base.socioeco.org/docs/01s_-_sse_event_span_ spanish_for_web_.pdf

Lemus, Blanca y David Barkin. Rethinking the Social and Solidarity Economy in Light of Community Practice. United Nations Research Institute for Social Development (2013). Acceso: 10 de setiembre de 2018. http://www.unrisd.org/80256B42004CCC77/(httpInfoFiles)/3262F14EE2B6C78AC1257B7200344026/\$fi le/Lemus $\% 20$ and $\% 20$ Barkin $\% 20 \mathrm{draft} \% 20$ paper.pdf

Leal Moya, Leticia. «Seguridad Humana. La responsabilidad de proteger» (2012). Acceso: 10 el setiembre de 2018. http://www.revistas.unam.mx/index.php/bmd/article/view/10626/9954

Martínez Osés, Pablo José. «Un análisis del poder en la agenda 2030: oportunidades para un cambio de paradigma de desarrollo». Economistas sin Fronteras. Dossieres EsF, n. ${ }^{\circ} 26$ (2017):11. Acceso: 10 de setiembre de 2018. http://base.socioeco.org/docs/dossieres-esf-26-repensando-el-modelo-de-sociedad-y-de-econom c3_ada.pdf

Naciones Unidas. «La Economía Social y Solidaria y el reto del Desarrollo Sostenible» (2014). Acceso: 10 de setiembre de 2018, http://unsse.org/wp-content/uploads/2014/08/Position-Paper_TFSSE_Esp1.pdf

Naciones Unidas. «La devastación de los pobres del mundo: El cambio climático amenaza los avances logrados en materia de desarrollo» (2017). Acceso: 10 setiembre de 2018. https://unchronicle.un.org/es/article/ la-devastaci-n-de-los-pobres-del-mundo-el-cambio-clim-tico-amenaza-los-avances-logrados-en 
Naciones Unidas. «La seguridad humana en las Naciones Unidas » (2012). Acceso: 10 de setiembre de 2018. https://www.unocha.org/sites/dms/HSU/Outreach/12-41684_Spanish\%20HUMAN\%20SECURITY\%20 Brochure_web_December\%2010.pdf

Porter, Michael E. y Scott Sterm, con Michael Green. Social Progress Index 2014 (2014). Acceso: 10 de setiembre de 2018. http://progresosocial.org.gt/wp-content/uploads/2014/12/resumen_ejecutivo_final.pdf

Porter, Michael E. y Scott Sterm, con Michael Green. «Social Progress Index 2015» (2015). Acceso: 10 de setiembre de 2018. https://www.socialprogressimperative.org/wp-content/uploads/2016/05/2015-SOCIALPROGRESS-INDEX_FINAL.pdf

Porter, Michael E. y Scott Sterm, con Michael Green. «Social Progress Index 2016» (2016). Acceso: 10 de setiembre de 2018. https://www.socialprogressimperative.org/wp-content/uploads/2016/06/SPI-2016-MainReport1.pdf

Porter, Michael y Scott Sterm. «Social Progress Imperative 2017» (2017). Acceso: 10 de setiembre de 2018. https://www.df.cl/noticias/site/artic/20170621/asocfile/20170621132906/spanish_2017_social_progress_index_report.pdf

Scott Sterm, Scott, Amy Wares y Sarah Orzell. Índice de Progreso Social 2017. Reporte Metodológico (2017): 9. Acceso: 10 de setiembre de 2018. http://www.socialprogressindex.com/assets/downloads/resources/es/ Spanish-2017-Social-Progress-Index-Methodology-Report.pdf

United Nations. Sustainable Development Goals. 17 Goals to transform our world (s.f.). Acceso: 10 de setiembre de 2018. http://www.un.org/sustainabledevelopment/ 